\title{
A Method of Building 3D Vector Model for Plant Root
}

\author{
Chen Fuqiang ${ }^{1}$, Zhou Xuecheng ${ }^{1,2}$, Yang Zishang ${ }^{1}$, Wang Gaofeng ${ }^{1}$ \\ ${ }^{1}$ College of Engineering, South China Agricultural University, Guangzhou City, China \\ ${ }^{2}$ South Agricultural Machinery and Equipment Key Lab of Ministry of Education, Guangzhou City, China
}

\section{Email address:}

15501840296@163.com (Chen Fuqiang), zxcem@scau.edu.cn (Zhou Xuecheng)

\section{To cite this article:}

Chen Fuqiang, Zhou Xuecheng, Yang Zishang, Wang Gaofeng. A Method of Building 3D Vector Model for Plant Root. Science Discovery. Vol. 5, No. 5, 2017, pp. 340-347. doi: 10.11648/j.sd.20170505.17

Received: June 22, 2017; Accepted: July 11, 2017; Published: August 7, 2017

\begin{abstract}
In order to achieve quantitative description and analysis of root architecture, a 3D vector model building method for plant root was proposed. Firstly, three data structures of root node, root branch and root system are designed. Secondly, according to the linear characteristic of root, the root skeleton points are extracted by the slice algorithm. Then, branch topological relations is established by using the path planning algorithm based on connectivity. Finally, the attribute parameters (radius, area, circumference, etc.) of the skeleton points are extracted by the method of branch slice. The results of the vector model are preserved in the three data structures of the root system, and the three-dimensional phenotypic detection of the root system is carried out using the model data. Through the analysis of the phenotypic test results, and compared with the manual measurement results, the verification of the proposed method of the feasibility and accuracy.
\end{abstract}

Keywords: Plant Root, Vector Model, Phenotypic Parameters

\section{一种植物根系三维矢量模型构建方法}

陈富强 ${ }^{1}$, 周学成 ${ }^{1},{ }^{2}$, 杨自尚 ${ }^{1}$, 王高峰 ${ }^{1}$

${ }^{1}$ 工程学院, 华南农业大学, 广州, 中国

2南方农业机械关键技术与装备教育部重点实验室, 广州, 中国

邮箱

15501840296@163.com(陈富强), zxcem@scau.edu.cn(周学成)

摘要: 为了定量描述和分析三维根系构型, 提出了一种植物根系三维矢量模型构建方法。首先为了满足算法需求设计 了根结点、根分支和根系统三个基本数据结构。其次，根据根系的线状特性采用横截面算法提取根系骨架点。然后利 用基于连通性的路径规划算法对根系骨架点分组建立分支拓扑关系。最后采用分支重切的方法提取骨架点的属性参数 以形成最终的根系矢量模型。利用该矢量模型完成根系的三维表型检测。通过与手工测量结果对比, 基本验证本文所 提出方法的可行性与准确性。根系矢量模型对于根表型参数的准确、自动、快速测量具有十分重要的意义。

关键词: 植物根系, 矢量模型, 表型参数

\section{1. 引言}

根系是植物的重要组成器官, 其形态构型决定了植物吸 收水分和养分的能力[1]。根系构型的检测与分析方法一直是
植物表型检测的重要研究内容, 而三维原位构型的自动检测 和分析方法是该领域目前亟待解决的一个技术瓶颈。根系矢 量模型作为三维表型参数测量的数据模型, 该模型的提出对 于根系表型参数的准确、自动和快速测量具有重要的意义。 
目前对植物根系测量与分析技术的研究大都基于二维图像 的根系平面构型完成, 专门针对原位根系的三维构型参数检 测与分析的研究则少见报道。其主要原因在于土壤等介质的 阻隔限制了对根系构型的直接观测, 以及根系本身的复杂性 和重叠性也加大了根系精确测量的难度[2-3]。目前三维根系 构型的相关研究工作主要集中在原位成像技术、图像处理和 三维可视化等方面, 对基于原位断层序列图像数据的利用则 大都停留在三维可视化阶段。为了实现原位根系三维构型参 数的无损检测, 笔者所在的课题组在国家自然科学基金和 “863”计划等科研项目的资助下，相继开展了基于XCT技术 的植物根系原位成像方法、CT序列图像的处理与分析方法、 原位根系的三维可视化重建方法。本文在此基础上设计了原 位根系三维矢量模型构建方法。并基于所构建的矢量模型实 现了根数、根长度、根体积、根表面积以及根夹角的自动化 测量。经过将多组根系样本的手工测量结果与本文方法的测 量结果作对比, 验证了根系矢量模型提取的准确性和稳定性。 对植物根系的定量研究有重要意义。

\section{2. 数据结构设计}

为了使本文提出的算法更加高效稳定的实现，根据根 系的形态特征和算法要求设计了算法的数据结构。本文算 法所需的数据结构包括: RootSlice、RootNode、RootBranch、 RootSystem四种基本结构, 分别代表根切片、根结点、根 分支和根系统。

\section{1. Root Node}

Root Node用来表示一个根结点, 是骨架模型和矢量 模型提取的关键数据结构。其保存了一些根结点的属性信 息。如图1所示:

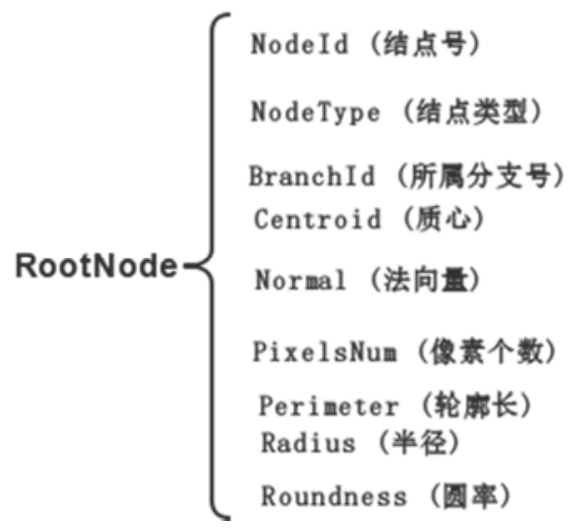

图1 RootNode属性。

其中结点类型根据结点在根上分布的位置不同分为 5 种类型, 如表1所示:

表 1 根结点类型。

\begin{tabular}{lll}
\hline $\mathbf{1}$ & NODE_TYPE_FIRST & 首结点 \\
\hline 2 & NODE_TYPE_LAST & 尾结点 \\
3 & NODE_TYPE_FORK & 分叉结点 \\
4 & NODE_TYPE_INFLECTION & 拐点 \\
5 & NODE_TYPE_NULL & 无类型 \\
\hline
\end{tabular}

\section{2. Root Slice}

RootSlice是一个根结点集合, 是骨架模型提取的关键 数据结构。其保存的是一张切片图像上所有的根结点信息。 切片图像的来源可以是原始的XCT序列图像, 也可以是对 三维实体模型重切得到的切面图像。RootSlice示意图如下:

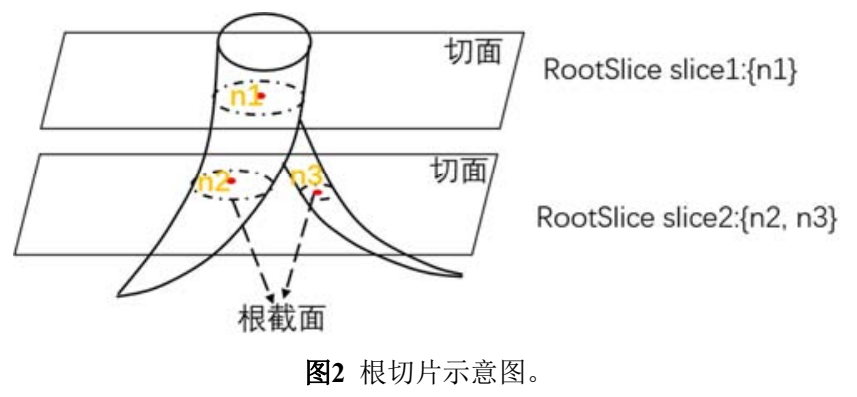

\section{3. Root Branch}

RootBranch用来表示一个根分支, 是矢量模型构建的 关键数据结构。其保存了一些根分支的属性信息。如图3 所示:

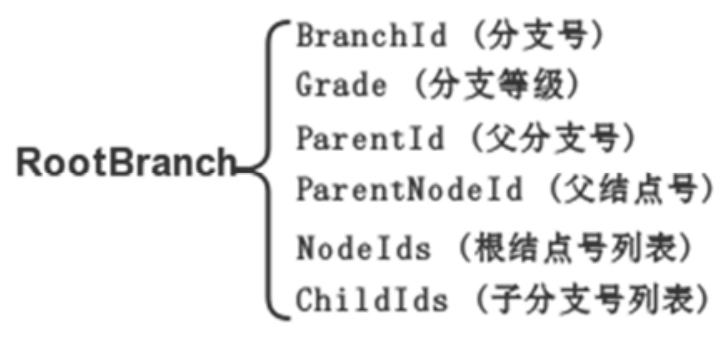

图3 RootBranch属性信息。

\section{4. Root System}

Root System用来表示一个整根, 其包含所有的根结点 (Root Node)和根分支(Root Branch)信息。同时还提供了很 多根结点或根分支的操作函数。用以辅助计算根的一系列 表型参数。Root System的常用操作如图4所示:

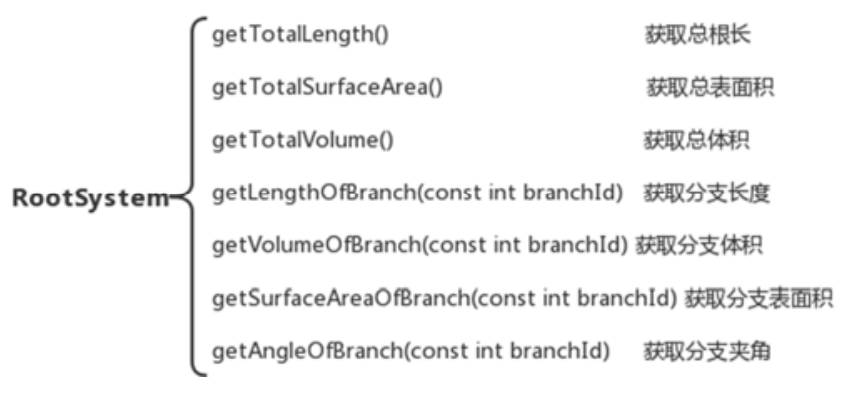

图4 根系统部分操作函数。

3. 矢量模型构建方法

算法流程图如图4所示: 


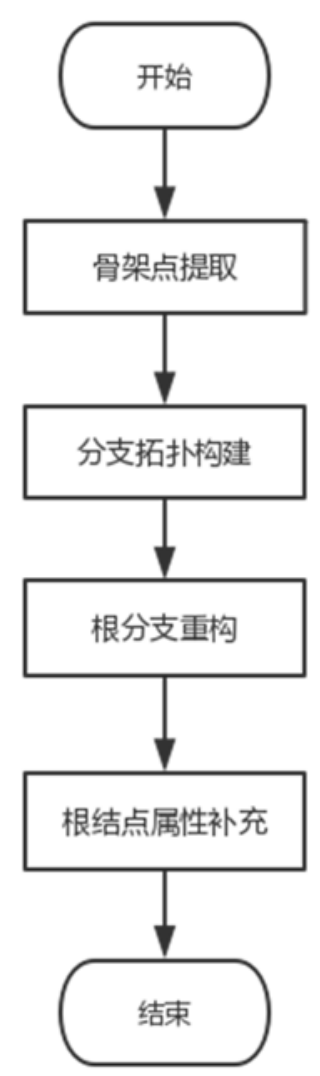

图5 算法流程图。

\section{1. 矢量模型}

为了更好的提取根系三维表型参数, 本文提出了一种 根系矢量模型。矢量模型是骨架模型的改进与拓展。其在 保持了骨架模型的平移旋转不变性、中心性、连通性、平 滑性和鲁棒性的基础上, 根据植物根系的形态特征拓展了 根分支拓扑关系(图8), 并以该拓扑关系为基础建立了根系 分层模型(图9)。同时矢量模型添加了根结点、根分支、根 系统等属性参数。矢量模型是根系表型参数测量的关键依 据。具体的属性数据见表格2所示:

表2 根属性数据。

\begin{tabular}{lll}
\hline & & 周长 \\
1 & 表面积 \\
& 结点属性 \\
& & 等效圆半径 \\
& 分支等级 \\
& & 父分支号 \\
& & 均值半径 \\
& 分支属性 & 体积 \\
& & 根长 \\
& & 根数量 \\
& & 根体积 \\
& & 根长度 \\
\hline
\end{tabular}

\section{2. 数据封装}

本算法所需要的数据源为三维实体模型。而由XCT层 析成像技术获取数据为 $\mathrm{CT}$ 序列图像。需将序列图像进行 封装。具体做法: 将CT序列图像按照顺序依次“堆叠“起来,
然后根据XCT数据采集时所设定的层间距设置相 邻两张 序列图像的间距。本文所使用的数据样本的层间距为 $0.08581081 \mathrm{~mm}$ 。封装示意图如图5:

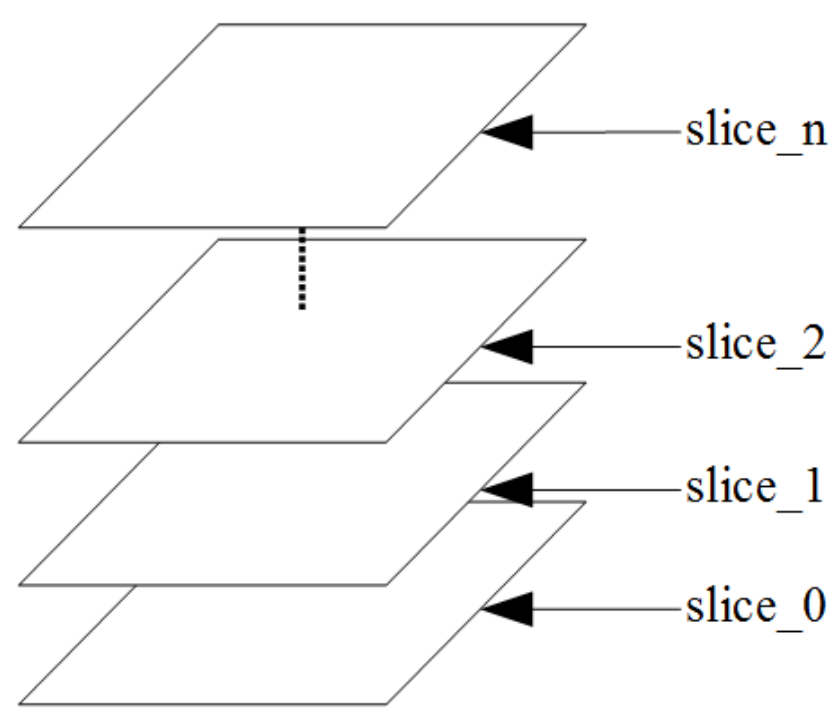

图6 数据封装示意图。

\section{3. 骨架点提取}

基于植物根系的柱体线状特性，本文采用了一种基于 横截面算法的三维骨架点提取方法 [3]。横截面算法是以 XCT序列图像为数据源。XCT图像可以看作是在固定位置、 固定方向下重切植物根系而得到的横截面, 图像中各个根 截面的质心基本近似为根系骨架点。通过提取每张CT图 像的根系骨架点, 得到根系的初始骨架点云。

由于根系表面较为粗粘以及存在一些包状凸起, 导致 会提取到一些伪骨架点(图6所示)。经分析发现, 伪骨架点 所在的截面面积一般较小, 此时可以通过设置面积阈值来 过滤伪骨架点。

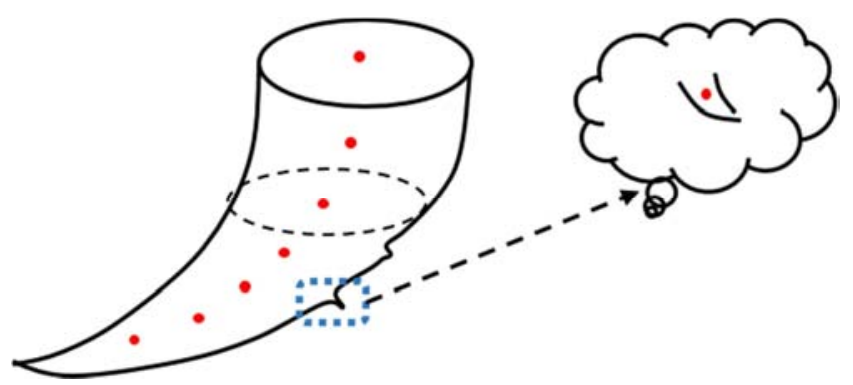

图7 伪骨架点示意图。

为了保留原始 $\mathrm{CT}$ 图像的Z向有序性, 本算法中使用了 一个RootSlice列表来保存提取到的骨架点。

\section{4. 分支拓扑构建}

通过上述步骤提取的骨架点只有空间几何关系, 没有 拓扑关系。本文根据根系的形态特征提出了一种分支拓扑 结构用来表示根分支之间的邻接关系。分支拓扑结构与树 状结构类似。图8展示一个根系分支拓扑关系的示意图, 
其中每个根分支被抽象成一个点, 分支与分支的邻接关系 用连接线来表示。

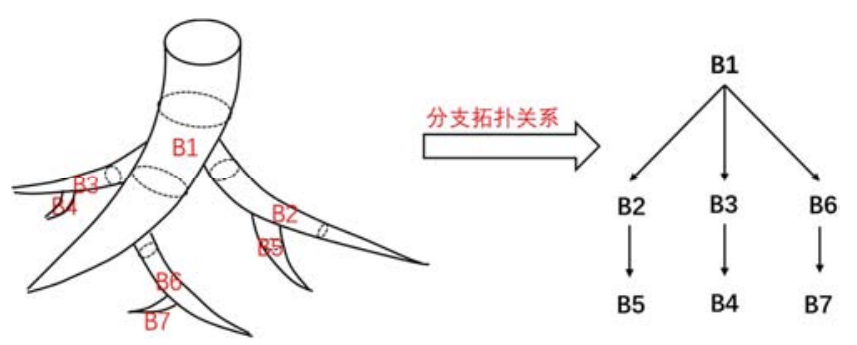

图8 分支拓扑关系示意图。

由分支的拓扑关系可以得到根系的层次模型，根据根 分支的邻接关系, 可以将根系看作是由不同层次级别的分 支所构成, 且层次内的各分支互不相连, 只与各自低层次 中的父分支相连。图9展示图8中根系的分级示意图, 该根 系一共有 1 个一级分支、 3 个二级分支、 3 个三级分支。

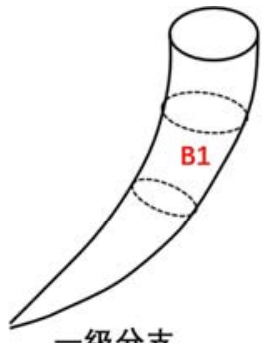

一级分支
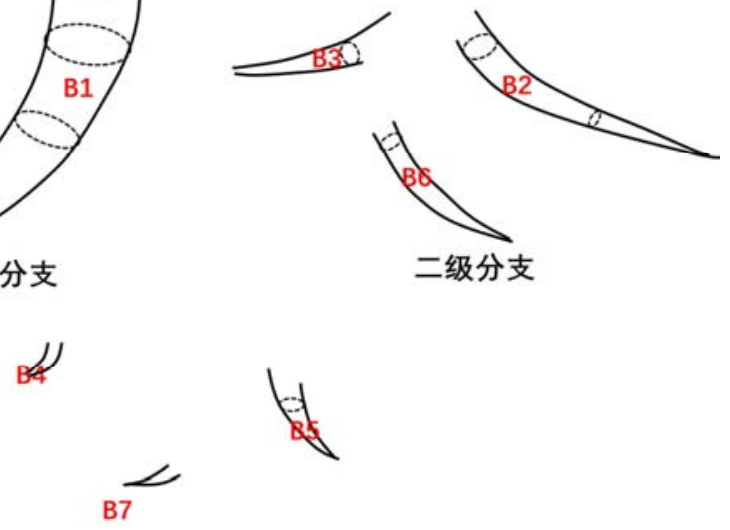

三级分支

图9 分支层次结构示意图。

为了建立上述描述的分支拓扑关系, 本文提出了一种 基于连通性的路径规划算法。该算法是以连通性分析为核 心思想, 并将面积变换率和偏转角以及最短路径作为进一 步的约束条件，来完成最后的分支拓扑关系构建。

连通性: 在两根结点中心之间作一直线段 $L_{\text {straight }}$, 若 该线段上的任意坐标点都落在根系体内, 则认为这两个根 结点是直连通的。

面积变化率: 如式 (1) 、式 (2) 和式 (3) 所示, 其中 $a r e a_{i}$ 和 $a r e a_{i+1}$ 分别是根结点 $\mathrm{i}$ 和根结点 $\mathrm{i}+1$ 的面积, rate $_{\text {area }}$ 即为面积变化率。

$$
\begin{gathered}
\text { area }_{\max }=\max \left(\text { area }_{i}, \text { area }_{i+1}\right) \\
\text { area }_{\min }=\min \left(\text { area }_{i}, \text { area }_{i+1}\right) \\
\text { rate }_{\text {area }}=\frac{\text { area }_{\max }-\text { area }_{\min }}{\text { area }_{\max }}
\end{gathered}
$$

偏转角: 如图5所示。图中 node $_{i-1} 、$ node $_{i}$ 和 $n o d e_{i+1}$ 为相邻的三个根结点。依次在相邻两个根结点之间连接直 线所形成的夹角 $\theta$ 即为偏转角。

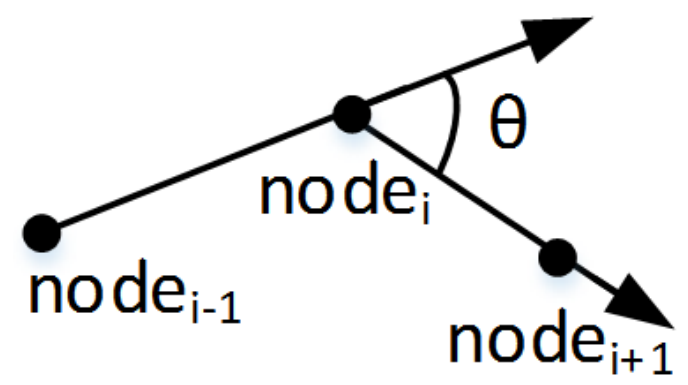

图10 偏转角示意图。

有了上述定义, 分支拓扑关系的提取步骤如下:

1. 遍历断层序列图像, 选取当前遍历的切片图像S1 和下一张切片图像 $\mathrm{S} 2$ 。其中 $\mathrm{S} 1$ 中得到的根结点为已分组结 点。S2中根结点为未分组结点。

2. 遍历 $\mathrm{S} 1$ 中的根结点, 计算和 $\mathrm{S} 1$ 中当前根结点 $\mathrm{n} 1$ 满 足连通性的 $\mathrm{S} 2$ 中的候选根结点集 $\mathrm{N} 2$, 当点集 $\mathrm{N} 2$ 的个数大 于 1 , 使用面积变化率篩选, 选取面积变化最小的根结点 作为当前分组的新结点加入其中。否则直接将点集N2中的 结点加入当前分组。重复该步骤直到S1中的结点全部遍历 过一遍。

3. 遍历 $\mathrm{S} 2$ 中的根结点, 选取还未分组的根结点 $\mathrm{n} 2$, 计算和 $\mathrm{n} 2$ 满足连通性的 $\mathrm{S} 1$ 中的候选根结点集 $\mathrm{N} 1$, 当点集 $\mathrm{N} 1$ 的个数大于 1 , 使用偏转角作为限制条件, 创建一个根 分支, 并选取偏转角最大的根结点作为新根分支的首结点。 否则创建一个根分支并直接把点集N1中的结点加入新根 分支。重复该步骤, 直到 2 中的结点全部遍历过一遍。

4. 重复1、2、3 步骤, 直到全部根切片遍历过一边结 束。

利用伪代码将分支拓扑构建过程做如下描述:

表3 分支拓扑构建伪代码描述。

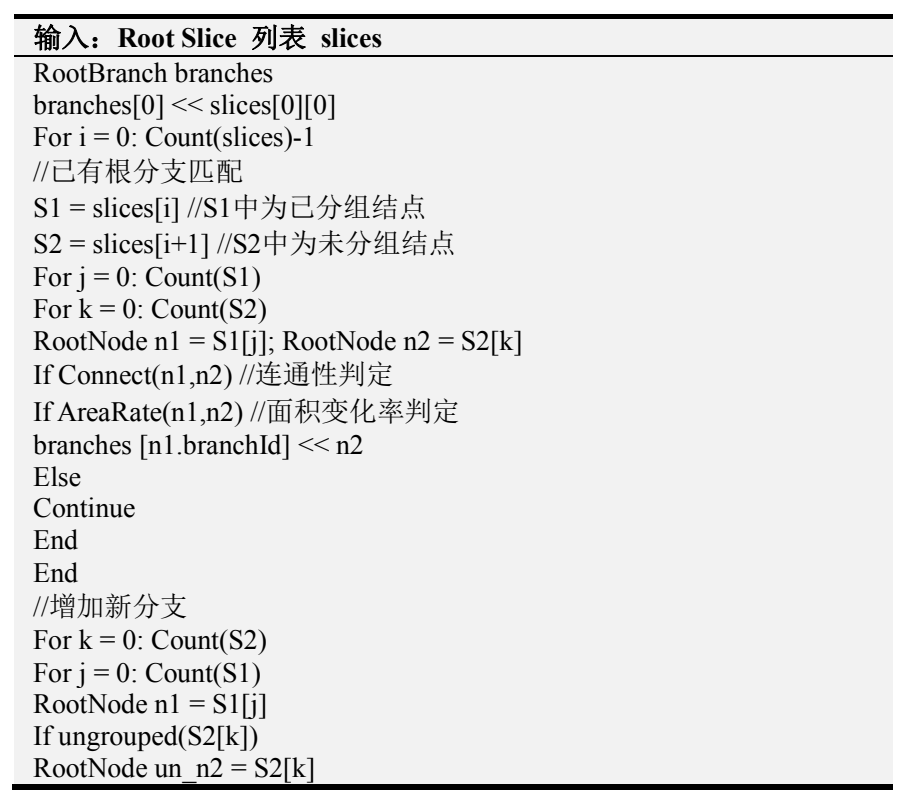




\begin{tabular}{l}
\hline 输入: Root Slice 列表 slices \\
\hline If Connect(n1,un_n2)//连通性验证 \\
If Angle(n1,un_n2)//偏转角验证 \\
creatBranch(branches,n1,un_n2) \\
Else \\
Continue \\
Else \\
Continue \\
End \\
End \\
End \\
输出: RootBranch列表 branches \\
\hline
\end{tabular}

使用颜色映射来显示提取到的分支骨架，最后得到的 分支拓扑关系如图10所示:

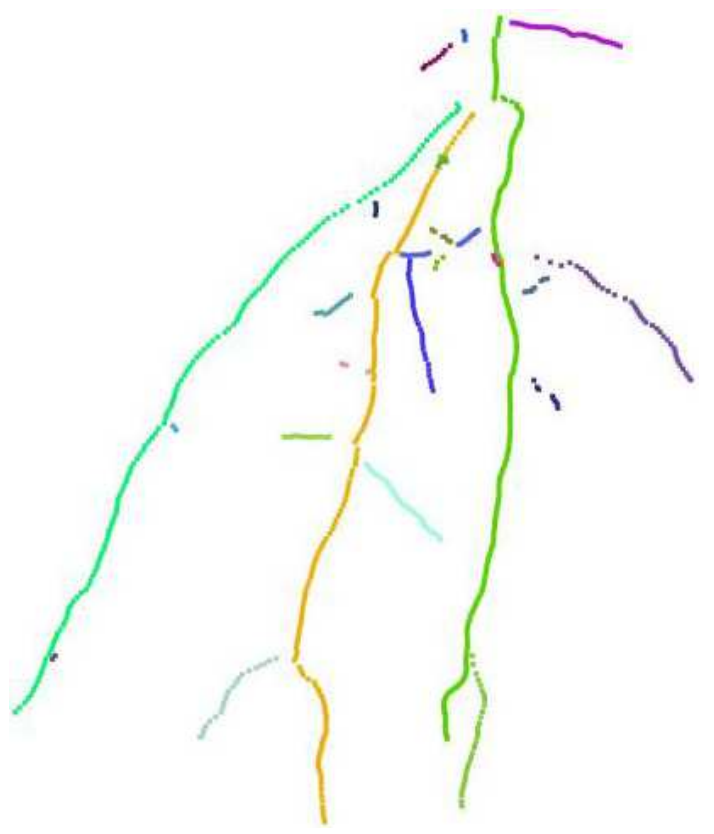

图11 分支拓扑提取效果。

\section{5. 分支重构}

虽然在骨架点提取过程中已经去除了杂质点影响。但 是提取到的根分支仍然存在部分结点偏移以及首位结点 缺失等问题, 需要对相关问题设计算法进行分支重构。

\section{5. 1. 根结点偏移}

对于处于分支分叉位置的根切片图像, 由于子分支的 影响, 父分支相关位置处的根截面质心与实际根结点位置 存在误差, 从而产生了根结点偏移现象(图11), 影响父分 支的骨架走向(图12)。

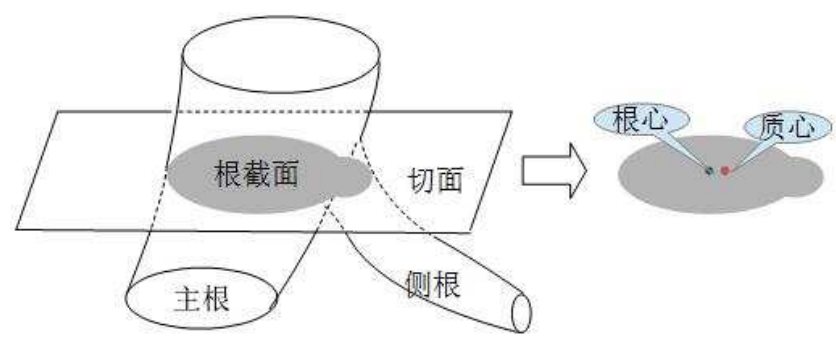

图12 根结点偏移现象。

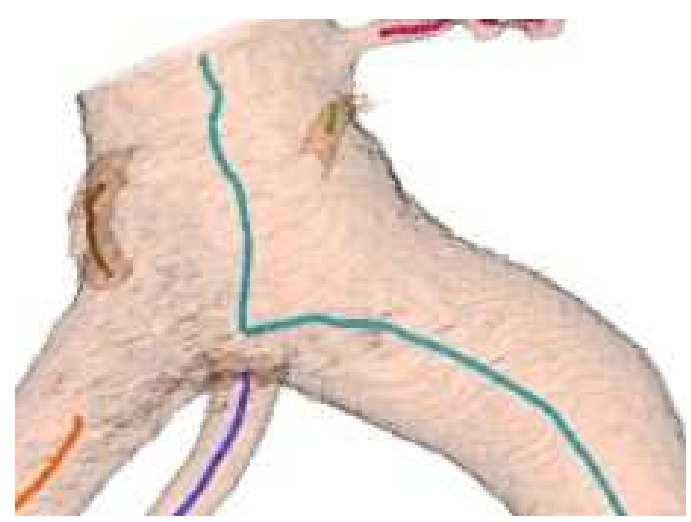

图13 骨架走向偏移。

经过分析发现, 根截面圆率越接近 1.0 , 其质心与实 际根结点位置误差就越小。分叉位置根截面由于受到子分 支影响, 其圆率值偏小。可以利用这个特性, 将分支偏移 处的根结点定位出来。剔除掉这些偏移根结点后, 然后使 用贝塞尔曲线拟合重新补充删除位置处的根结点。其纠正 效果如下:

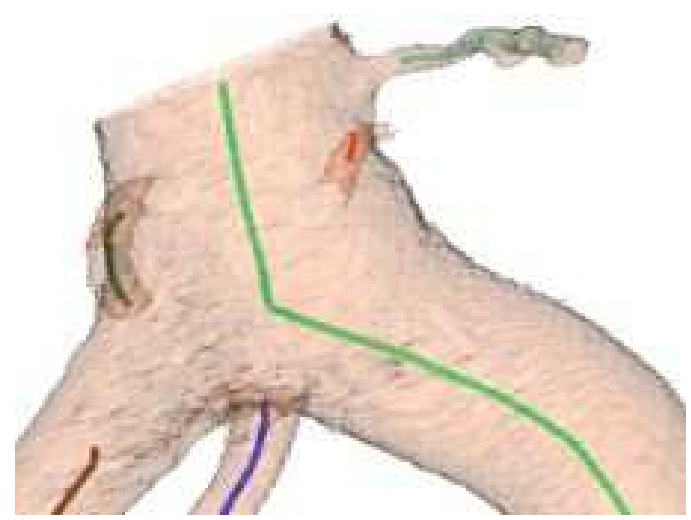

图14 拟合纠正效果。

\section{5.2. 分支父结点偏移}

在构建分支拓扑关系时子分支的父结点是利用结点 之间的连通性求得的, 这样获取的父结点位置可能存在偏 差现象, 图14中描述了这种偏移现象, 由于连通性约束分 支父结点会被划分到 $\mathrm{P} 2$, 而实际的父节点位置为 $\mathrm{P} 1$ 。需要 重新寻找子分支父结点。

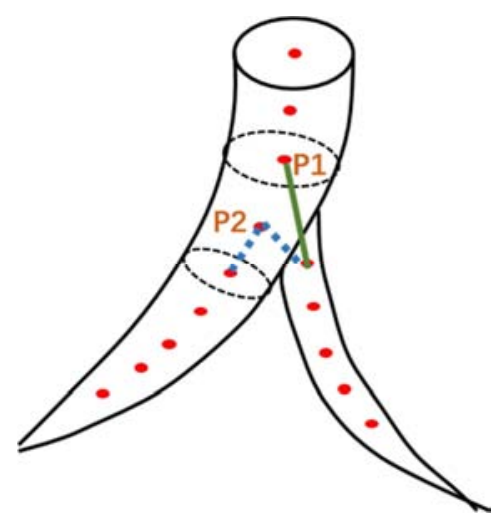

图15 父结点位置偏移示意图。 
重新确定子分支父结点, 其算法步骤如下:

1. 在父分支中找到所有与子分支首结点具有连通性 的结点作为候选分支父结点。

2. 子根分枝首根结点 $\mathrm{a}$ 到上述候选分支父结点 $\mathrm{b}$ 构成 一条直线。计算此直线的方向与子根分枝首根结点法向量 之间的夹角 $\theta$, 然后选择使得 $\theta$ 最接近零所对应的候选分 叉根结点作为最终分叉根结点。

分支父结点纠正效果如图15所示:

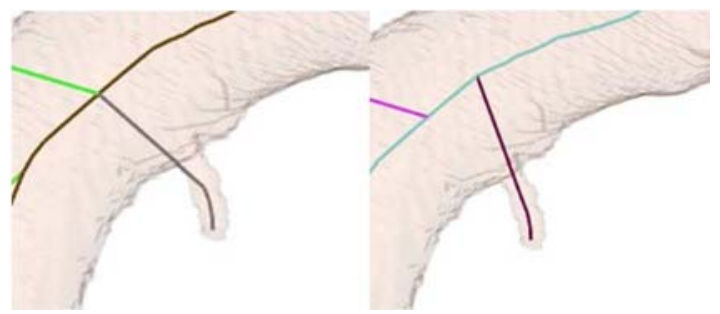

图16 分支父结点纠正效果。

\section{5. 3. 分支端点补充}

由于在提取根结点时相邻切面之间具有一定间距, 并 且在分支分叉位置受到父分支的干扰导致子分支首段位 置缺少结点信息。因此子根分枝与父根分枝之间存在一定 的间隙, 根分枝的末端结点与实际根尖之间也有一定间隙 （图17）。

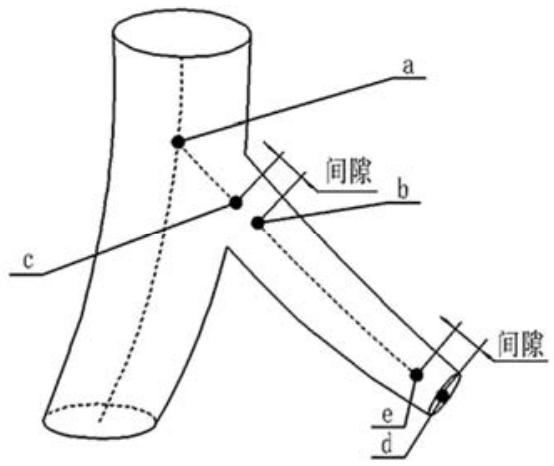

图17 根分支间隙示意图。

为了保证根系骨架完整性, 同时不影响后续根系参数 测量的精度。需要对各个根分支的端点进行结点补充。具 体补充方法如下:

1）补充末端根结点

补充末端根结点, 即在子根分枝远离父分枝的一端补 充若干根结点, 具体步骤如下:

(1)在三维空间中作一条射线, 起点为子根分枝的末端 根结点中心 (图17的根结点e), 方向为该根结点法向量;

(2)设该射线与根分枝表面的交点为 $\mathrm{d}$, 则得到线段 $e d$

(3)在线段 $e d$ 上等间距取若干点作为补充根结点的中 心，新根结点的法向量设置为该线段的方向向量。

2) 补充首端根结点

补充首端根结点, 即在子分支靠近父分支一段补充若 干结点。具体步骤如下:

(1)在图3.29a分叉根结点 $\mathrm{a}$ 与子根分枝首根结点 $\mathrm{b}$ 之间 作一空间直线段 $a b$, 该直线段与父分支表面的交点记为 $\mathrm{c}$;
(2)在线段 $c b$ 上等间距取若干点作为补充根结点的中 心，新根结点的法向量设置为该线段的方向向量。

分支端点补充效果如图18所示, 分图 $\mathrm{a}$ 是为补充效果。 分图 $\mathrm{b}$ 是补充后的效果。

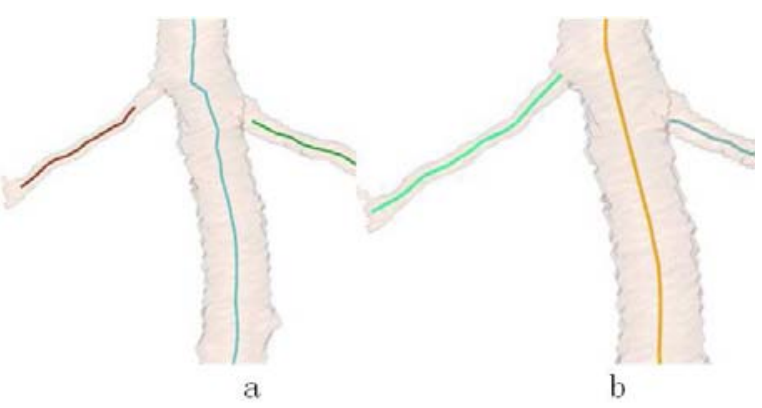

图18 分支端点补充效果。

\section{6. 根结点属性补充}

经过前述步骤已将获取到一个完整的带有分支拓扑 关系的骨架模型。但该模型并不能满足根系表型参数测量 的要求。需要在骨架模型基础上补充属性参数来形成最终 的矢量模型, 该模型是根表型参数测量的关键。

为了补充属性参数, 需要进行分支重切来补充参数信 息(图18)。该过程需要三维实体数据作为数据源, 所以先 将XCT序列图像进行数据封装。具体分支重切步骤如下:

1. 从根系统中获取一个根分支，向下遍历分支中的 每一个根结点。获取当前结点的位置坐标, 将其设置为切 面中心。获取当前结点的法向量, 将其设置为切面法向量。 此时切面已位于当前结点位置且垂直于根分支生长方向。

2. 以当前结点 $\mathrm{a}$ 为起点做一条垂直于结点法向量的射 线, 该射线与根分支表面交与点 $b$. 将线段 $a b$ 的距离记为 该结点的等效圆半径 $\mathrm{R}$, 由于根截面形状是类圆形, 为了 保证切片完全包含该根截面, 根据实验分析设置一个半径 调整系数 1.1 , 将切面半径设置为 $\mathrm{R} * 1.1$ 。

3. 使用设置好方向大小的切面重切当前结点, 将提 取到的根截面的周长面积属性值赋值给当前根结点。

4. 遍历完分支所有根结点后, 将计算得到的分支长 度、体积属性值赋值给当前根分支。

5. 遍历完根分支后，将计算得到的根长、根体积、 根数等信息赋值给根系统。

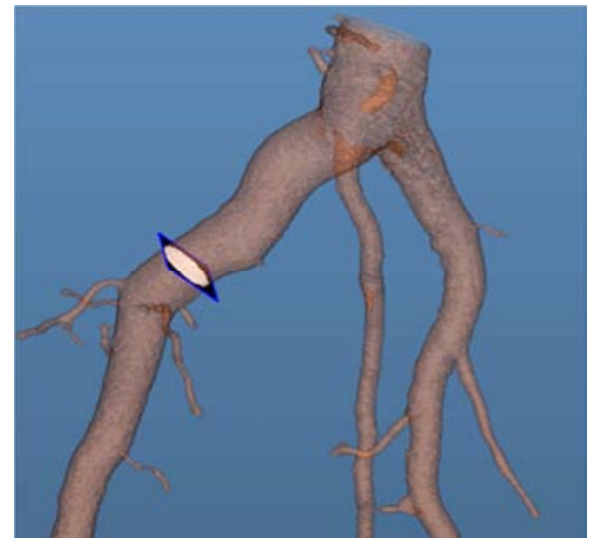

图19 分支重切示意图。 


\section{4. 结果与分析}

在windows平台上利用可视化工具包VTK和医学图像 分割与配准工具包ITK实现上述算法。通过将三维重建模 型与矢量模型进行对比, 并将基于矢量模型提取的参数测 量结果与手工测量结果作对比来验证本文算法的可行性 与有效性。

为获取对比数据, 本文采用了一系列传统方法来获取 根构型参数。如: 采用排沙法获取根系体积; 采用染色液 蘸根法获取根系表面积。采用直接截获法获取根系总长度。

本文采用了两组测试样本, 分别是是生长在有机基质 中的榕树和栃子幼苗根系图像。图像由工业CT设备获取。 主要扫描参数为: 管电压 $80 \mathrm{kv}$, 管电流 $1 \mathrm{~mA}$ 。经过扫描, 获取到分辨率为 $512 \times 512$ 的 16 位断层序列图像。对原位 CT 序列图像经过适当滤波、插值和三维封装预处理后用 于后续算法的数据源。

实验的第一组数据为榕树苗。对其序列图像进行三维 重建。重建采用移动立方体法。设置其等值面参数为 128 。 重建完成后对模型进行孔洞修补, 设置其孔洞填充最小尺 寸参数为 10 。最后生成的三维重建模型, 利用本文所提出 的算法提取榕树苗矢量模型。两种模型的叠加效果如图19。 其中浅黄色为三维重建结果。绿色分支为被拾取到的矢量 模型的根分支。各个分支骨架使用不同颜色映射表示。

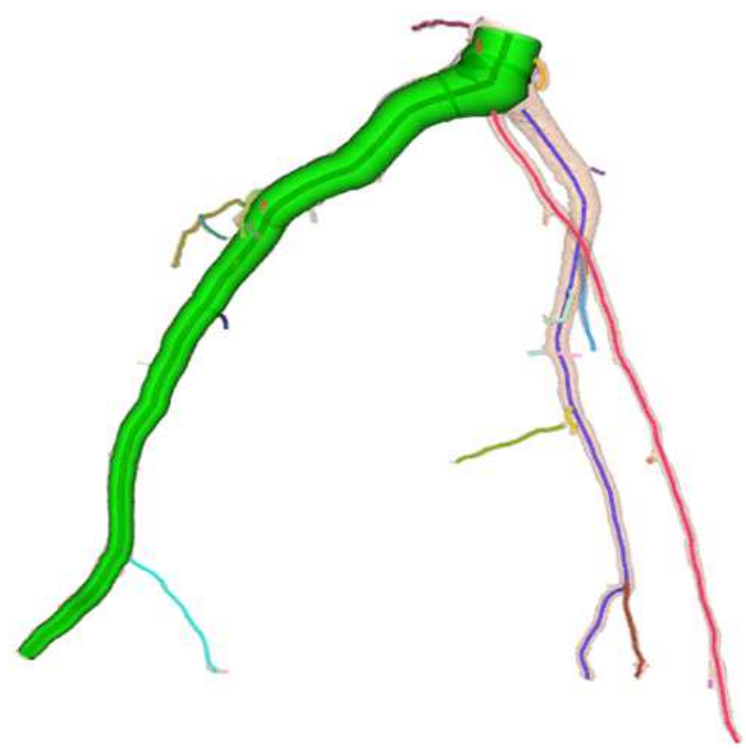

图20 叠加效果。

基于矢量模型所测量的根系三维表型参数值与对应 手工测量值如表4所示。由表可知，根分枝数、根总长、 根表面积以及根体积的测量误差分别为 $0 \% 、 2.1 \% 、 6.26 \%$ 、 $1.02 \%$ 。

表4 榕树苗参数测量结果对比分析。

\begin{tabular}{llll}
\hline 三维构型参数 & 系统测量值 & 手工测量值 & 误差 \\
\hline 分枝数 & 25 & 25 & $0 \%$ \\
总长度 & $611.48 \mathrm{~mm}$ & $624.60 \mathrm{~mm}$ & $2.10 \%$ \\
总表面积 & $4887.41 \mathrm{~mm}^{2}$ & $4599.38 \mathrm{~mm}^{2}$ & $6.26 \%$ \\
总体积 & $5320.25 \mathrm{~mm}^{3}$ & $5374.81 \mathrm{~mm}^{3}$ & $1.02 \%$ \\
\hline
\end{tabular}

本次实验的另外一组样本是栃子幼苗, 对数据进行三 维封装、分割、重建并进行矢量模型提取, 获得的叠加效 果如下图20。

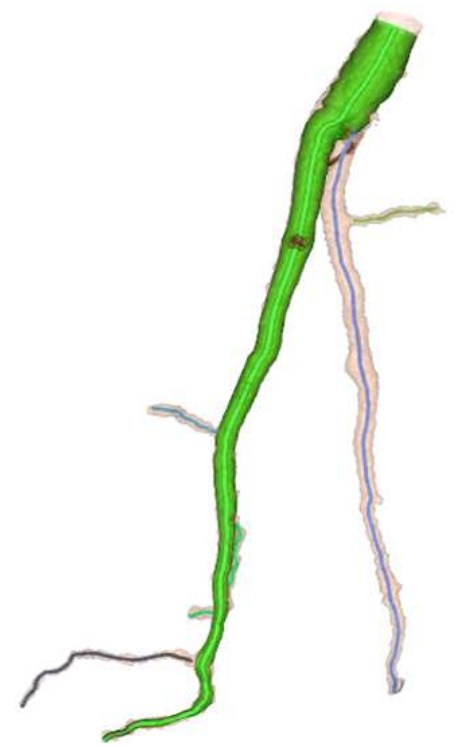

图21 叠加效果。

基于矢量模型所测量的根系三维表型参数值与对应 手工测量值如表 5 所示。由表可知，根分枝数、根总长、 根表面积以及根体积的测量误差分别为 $0 \% 、 4.1 \% 、 2.91 \%$ 、 $1.68 \%$ 。

表5 㭛子树苗参数测量结果对比分析。

\begin{tabular}{llll}
\hline 三维构型参数 & 系统测量值 & 手工测量值 & 误差 \\
\hline 分枝数 & 8 & 8 & $0 \%$ \\
总长度 & $367.67 \mathrm{~mm}$ & $352 . .97 \mathrm{~mm}$ & $4.10 \%$ \\
总表面积 & $8757 . .45 \mathrm{~mm} 2$ & $9011.86 \mathrm{~mm} 2$ & $2.91 \%$ \\
总体积 & $20976 . .92 \mathrm{~mm} 3$ & $21335 . .15 \mathrm{~mm} 3$ & $1.68 \%$ \\
\hline
\end{tabular}

由上述测试与分析结果可知, 本文提出的根系矢量模 型构建方法的有效性和可行性。系统参数测量结果与手工 测量结果有一定误差主要因为根系表面较为粗䊁造成了 伪根结点。后续将继续完善伪根结点剔除算法以进一步提 高测量精度。

\section{5. 结论}

本文介绍了植物根系三维矢量模型的提取方法。并以 此模型为依据实现了原位根系构型参数的自动测量。通过 实验数据测试证实了该方法的可行性与有效性。

\section{参考文献}

１1］罗锡文, 周学成, 严小龙. 植物根系三维构型原位观测技 术的研究进展[C]. 中国农业工程学会2005年学术年会论文 集, 2005:421-425。 
[2]罗锡文, 周学成, 严小龙, 等. 基于XCT技术的植物根系 原位形态可视化研究 $[\mathrm{J}]$. 农业机械学报. 2004，35(2): 104-133。

[3] 张常玲, 周学成, 李骈臻. 土壤中植物根系的层析成像检 测试验 $[J]$. 光学应用: 中英文版, 2014(2):19-24。

[4] 田绪红, 李志垣, 韩国强等. 基于横截面算法的三维植物 根系图象骨架生成方法 $[\mathrm{C}] / /$ 第十二届全国图象图形学学术 会议论文集. 2005:655-658。

[5] Clark, Randy T, Mac Curdy, Robert B, Jung,Janelle K, et al. Three-Dimensional Root Phenotyping with a Novel Imaging and Software Platform [J]. PLANT PHYSIOLOGY. 2011, 156(2):455-465.

[6] Stefan Mairhofer,Saoirse R. Tracy, et al. RooTrak: Automated Recovery of Three-Dimensional Plant Root Architecture in Soil from X-Ray Microcomputed Tomography Images Using Visual Tracking [J]. PLANT PHYSIOLOGY, 2012, 158(2):561-569.

[7] DD Van, R Metzner, J Kochs, JA Postma, D Pflugfelder et al. Quantitative 3D Analysis of Plant Roots Growing in Soil Using Magnetic Resonance Imaging [J]. Plant Physiology, 2016, 170(3):1176-1188.

[8] 张晓东, 罗火灵. VTK图形图像开发进阶[M]. 北京: 机械 工业出版社，2015:170-192。
[9] 周振环, 郑小中, 赵明. 三维图像编程实验 $[\mathrm{M}]$. 北京: 电 子工业出版社, 2011:82-86。

[10] Heeraman D A, Hopmans J W, Clausnitzer V. Three dimensional imaging of plant roots in situ with X-Ray computed tomography [J]. Plant and Soil, 1997, 189(2):167-179.

[11] 胡占利, 基于VTK的医学图像三维重建及交互研究[D]. 哈 尔滨：哈尔滨工程大学，2008。

[12] 陈郁洤, 周学成, 罗锡文, 等. 基于ITK和VTK的原位根 系三维可视化研究 [C]//中国农业工程学会2011年学术年会 论文集，2011:1164-1168。

[13] Kuijken R C, van Eeuwijk F A, Marcelis L F, et al. Root phenotyping: from component trait in the lab to breeding [J]. Journal of Experimental Botany, 2015, 66(18): 5389-5401.

[14] 李骈臻, 周学成, 张常玲, 等. 基于骨架模型的植物根系 三维构型可视化方法 [J]. 计算机工程与设计, 2014, 35(11):3913-3917。

[15] Metzner Ralf, Eggert Anja, van Dusschoten, Dagmar et al. Direct comparison of MRI and X-ray CT technologies for 3D imaging of root systems in soil: potential and challenges for root trait quantification [J]. Plant Methods, 2015, 11(1):17. 